\title{
Entérobactéries productrices de carbapénémases en médecine intensive : thérapeutique
}

\author{
Management of Carbapenemase-Producing Enterobacteriaceae in Intensive Care Unit
}

\author{
J.Y. Mootien · J.-R. Zahar \\ Reçu le 22 juin 2018; accepté le 26 juin 2018 \\ (c) SRLF et Lavoisier SAS 2018
}

Résumé Les infections à entérobactéries productrices de carbapénémases peuvent représenter une pathologie redoutable, notamment dans les situations cliniques graves, en raison des possibilités thérapeutiques limitées. En France, les mécanismes OXA-48 et OXA-48-like (78\%) sont les plus fréquemment retrouvés. Les stratégies thérapeutiques actuelles limitées ont mis en exergue l'intérêt de certaines vieilles molécules et des associations d'antibiotiques avec une optimisation de leurs modalités d'administration. Dans l'attente de l'apport des futures options thérapeutiques, les essais contrôlés randomisés sont plus que nécessaires. Nous devons nous inspirer de l'expérience de ceux qui prennent en charge ces infections. La maîtrise du bon usage des antibiotiques reste toujours d'actualité afin de préserver l'efficacité des molécules existantes.

Mots clés Entérobactéries productrices de carbapénémases · Bactéries multirésistantes - Stratégie de prévention ·

Association d'antibiotiques · Nouvelles options thérapeutiques

Abstract Infections due to carbapenemase-producing ente-
robacteriaceae may represent a potential threat due to the
scarcity of the available therapeutic resources. OXA- 48 and

J.Y. Mootien $(\bowtie)$

Service de réanimation médicale,

unité de conseil en antibiothérapie,

groupe hospitalier région Mulhouse et Sud-Alsace,

20, avenue du Docteur-René-Laennec,

F-68100 Mulhouse, France

e-mail : mootieny@ghrmsa.fr

J.-R. Zahar

IAME, UMR 1137, université Paris-XIII,

Sorbonne-Paris-Cité, France

Département de microbiologie clinique,

unité de contrôle et de prévention du risque infectieux, groupe hospitalier Paris-Seine-Saint-Denis, AP-HP,

125, rue de Stalingrad, F-93000 Bobigny, France
OXA-48-like are most prevalent in France. Older agents have become the last resort choices as treatment options are relatively few and combination therapy with optimal administration should be considered. Therapeutic future options as well as randomized controlled trials are needed. Meanwhile, we need to look at experiences of those managing this health problem and promoting better use of antibiotics stands as a sheer necessity.

Keywords Carbapenemase-producing Enterobacteriaceae · Multidrug-resistant bacteria - Infection control measures · Combination therapy $\cdot$ Future therapeutic options

\section{Introduction}

La résistance aux carbapénèmes est un phénomène préoccupant pouvant comporter un taux de mortalité non négligeable dans certaines infections [1]. À ce jour, aucune recommandation thérapeutique concernant cette problématique n'a été émise. Le mécanisme de résistance est double : soit par la production de carbapénémases, soit par la production de bêtalactamases à spectre élargi et/ou une céphalosporinase de type Amp $\mathrm{C}$ associée à une diminution de la perméabilité membranaire. Les différents types de carbapénémases ont été abordés dans le chapitre précédent, et nous traiterons dans cet article les différentes possibilités thérapeutiques envisageables dans le cadre des infections à entérobactéries productrices de carbapénémases (EPC) après avoir réalisé une revue de la littérature. Les premiers cas d'EPC sont apparus en France au cours des années 2000, caractérisés par un lien quasi constant avec une hospitalisation initialement à l'étranger [2]. Selon le bilan épidémiologique national publié par Santé publique France en fin décembre 2015, on constate une augmentation du nombre d'épisodes concernant les EPC [3]. L'espèce Klebsiella pneumoniae est la plus impliquée (58\% des épisodes), suivie d'Escherichia coli (36\%) et d'Enterobacter cloacae (12\%). En France, les 
mécanismes OXA-48 et OXA-48-like (78 \%) sont les plus fréquemment retrouvés, suivis du mécanisme NDM (14\%) et de KPC (6\%), selon ce rapport.

Nous aborderons dans un premier temps les différentes familles d'antibiotiques ou de molécules utilisées, puis les associations envisageables et enfin les perspectives thérapeutiques, avant de proposer un arbre thérapeutique dans les infections sévères ainsi qu'un schéma d'antibiothérapie en fonction du type de carbapénémase (Fig. 1, Tableau 1).

\section{Carbapénèmes}

Envisager les carbapénèmes comme possibilité thérapeutique d'une bactérie productrice d'une carbapénémase peut paraitre absurde d'un premier abord. Cela mérite certaines explications. Tout d'abord, comme souligne le CASFMEUCAST [4] (Tableau 2), les concentrations critiques des carbapénèmes ont été définies de sorte que les isolats cliniques incluant la majorité des carbapénémases soient catégorisés « intermédiaires » ou « résistants » aux carbapénèmes. Certains isolats producteurs de carbapénémases peuvent aussi être catégorisés " sensibles » à cette classe thérapeutique. La présence d'une carbapénémase n'interfère donc pas sur la catégorisation de ces EPC, et la présence d'une carbapénémase n'exclut en aucun cas, de façon définitive, l'utilisation des carbapénèmes qui restent une option thérapeutique sous certaines conditions.

La CMI conditionne les possibilités de succès de ce pari thérapeutique. Elle est variable dans ce contexte en fonction du taux d'expression de la carbapénémase et de la coexistence d'autres mécanismes de résistance tels qu'une BLSE ou encore une imperméabilité membranaire. Daikos et Markogiannakis [5] rapportent des résultats très intéressants obtenus chez l'animal ainsi que chez l'homme quand la CMI des carbapénèmes était inférieure ou égale à $4 \mathrm{mg} / \mathrm{l}$ dans les infections à K. pneumoniae KPC (KP-KPC). Le taux de survie dans les associations thérapeutiques comportant le méropénème passait de 87,75 à $65 \%$ si la $\mathrm{CMI}$ variait respectivement de 4,8 et $16 \mathrm{mg} / 1$ [6]. Les stratégies thérapeutiques incluant un carbapénème comportaient le taux de mortalité le plus faible (18,8\%), alors que dans les options excluant un carbapénème il passait à 30,7\% [7].

On note également une augmentation de la mortalité de 19,3 à $35,5 \%$ dans les schémas comportant l'association d'un carbapénème à un autre antibiotique sensible si toutefois la CMI devenait supérieure à $8 \mathrm{mg} / 1$ [8]. Ainsi, Tumbarello et al. [9] proposent également de privilégier l'utilisation d'un carbapénème dans les infections à KP-KPC si toutefois la CMI est inférieure ou égale à $8 \mathrm{mg} / \mathrm{l}$, alors que d'autres études retrouvent des résultats encore intéressants avec une CMI inférieure ou égale à $16 \mathrm{mg} / \mathrm{l}$ dans le cadre d'une triple association [6]
Les modalités d'administration des carbapénèmes permettant une optimisation des paramètres pharmacodynamiques de cette classe thérapeutique $(\mathrm{T}>\mathrm{CMI}=50 \%)$ potentialisent également l'efficacité de ces molécules $[5,10]$. Les probabilités d'atteindre cette valeur cible sont respectivement de 69,93 et $100 \%$ pour une perfusion de $1 \mathrm{~g}$ (30 minutes), $1 \mathrm{~g}$ ( 3 heures) et $2 \mathrm{~g}$ ( 3 heures) sur des souches ayant une CMI du méropénème à $4 \mathrm{mg} / 1$ [11].

Une association de deux carbapénèmes est proposée par certains travaux $[12,13]$. L'utilisation de l'ertapénème avec une forte dose de méropénème ou de doripénème est une stratégie possible dans les infections à KP-KPC comportant une CMI des carbapénèmes élevée ou dans les situations de résistance à la colistine [14-16]. L'ertapénème ayant une meilleure affinité pour les carbapénémases agit comme un substrat suicide. Il bloque l'enzyme et permet ainsi l'action de l'autre carbapénème.

Bassetti et al. [17] proposent dans les infections comportant une CMI inférieure ou égale à $8-16 \mathrm{mg} / \mathrm{l}$ l'administration de méropénème à fortes doses en perfusion prolongée, associé à deux autres antibiotiques actifs in vitro. Dans le cas contraire, une stratégie comportant l'association de trois autres molécules actives in vitro excluant les carbapénèmes doit être privilégiée.

$\mathrm{Au}$ total, les carbapénèmes restent une option thérapeutique essentielle si toutefois les valeurs de CMI décrites ciavant sont respectées. Leur combinaison avec d'autres molécules actives in vitro comporte le taux de mortalité le plus faible parmi les différentes associations proposées [7].

\section{Colistine}

La colistine est utilisée en clinique à partir de 1959 puis délaissée dans les années 1970 en raison de sa néphrotoxicité. L'émergence de bacilles à Gram négatif (BGN) multirésistants a conduit à reconsidérer cette molécule en thérapeutique.

La colistine est administrée sous forme d'une prodrogue inactive (colistiméthate). En raison de sa conversion lente en forme active, il est recommandé d'administrer une dose de charge afin d'atteindre les taux thérapeutiques dans les plus brefs délais. Ces taux sont atteints en 12 à 24 heures lors de l'utilisation d'une dose de charge, alors que plus de 48 heures seront nécessaires dans le cas contraire $[11,18]$.

La variation interindividuelle de la concentration et la fenêtre thérapeutique étroite démontrent l'intérêt de monitorer les concentrations sériques. Le risque de toxicité rénale connue de la colistine est majoré en cas d'utilisation de fortes doses et d'association de médicaments néphrotoxiques. Dans le cadre de la prise en charge des patients de réanimation, l'optimisation de l'hydratation peut permettre de diminuer ce risque $[19,20]$. Par ailleurs, des sous-dosages 


\section{I) Pneumonies, Bactériémies, Infections intra-abdominales.}

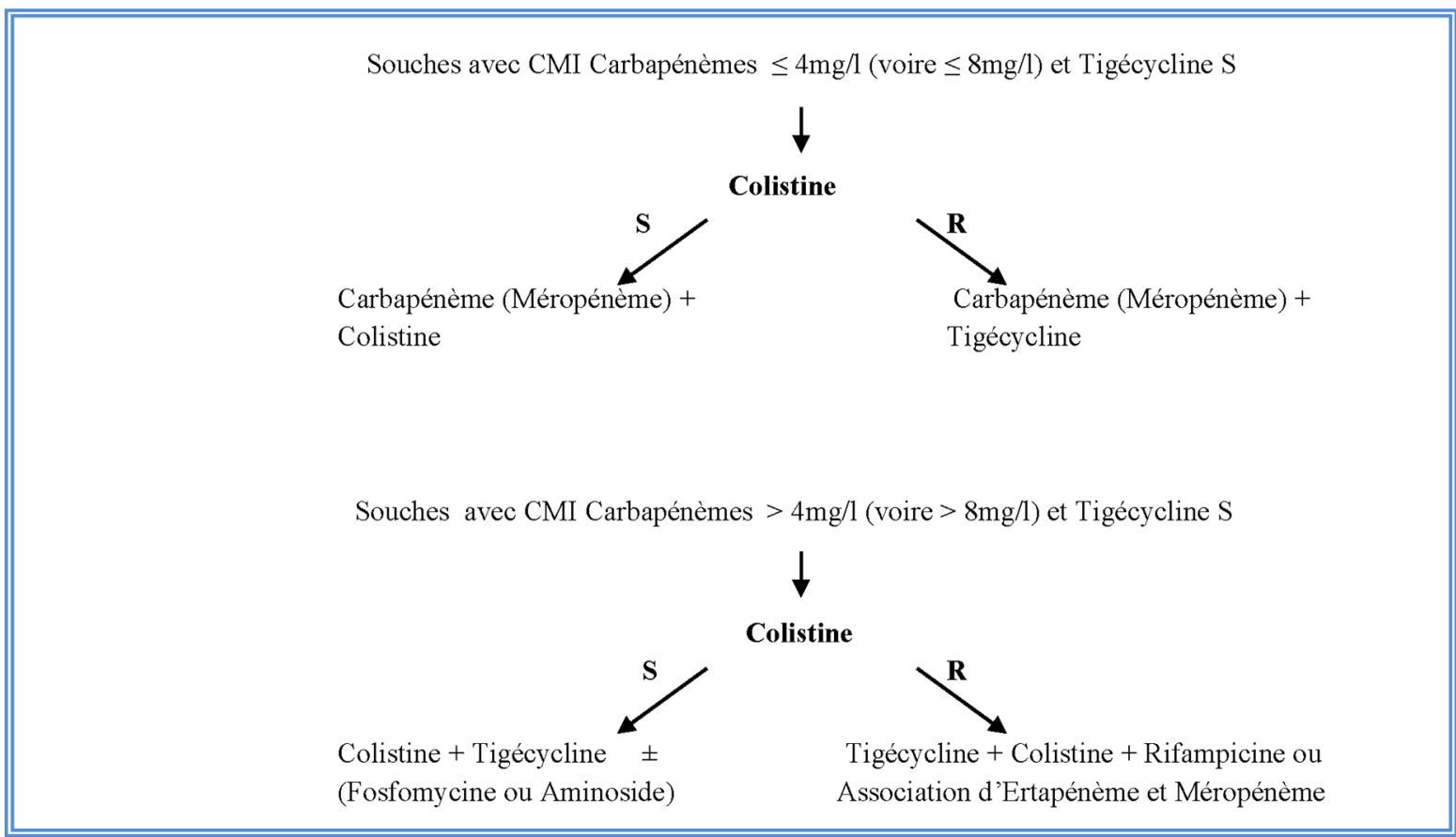

\section{II ) Infections des voies urinaires sévères}

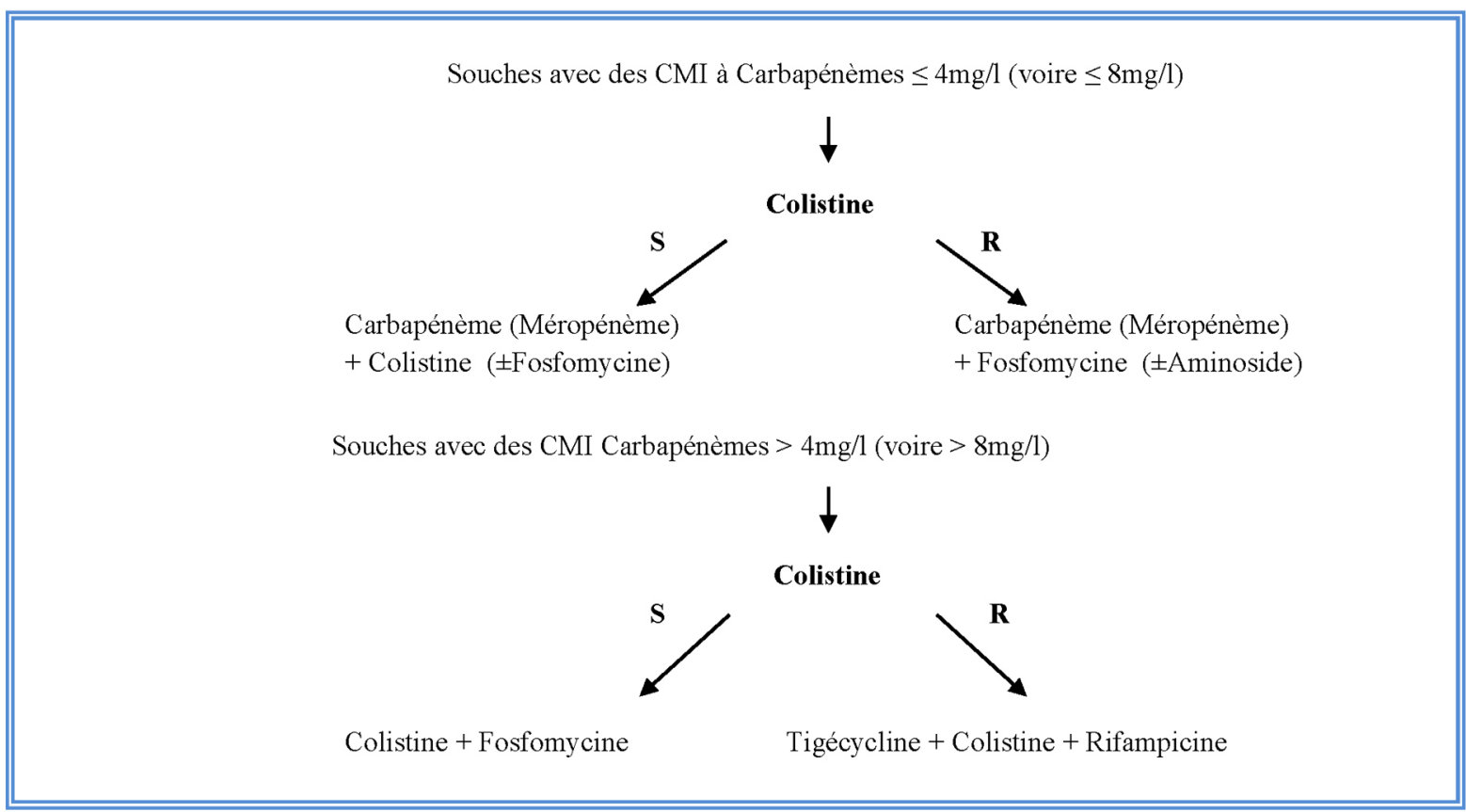
(R) Résistant
(S) Sensible

(i) Dans ces différentes infections une stratégie par Ceftazidime - avibactam pourrait être envisagée, en cas d'impasse thérapeutique [17]

(ii) Attention à la fonction rénale en cas d'association Colistine et d'un Aminoside

Fig. 1 Arbre thérapeutique proposé 
Tableau 1 Schéma d'antibiothérapie proposé en fonction du type de carbapénémase

\begin{tabular}{|ll|}
\hline KPC-carbapénémase & $\begin{array}{l}\text { Méropénème }+ \text { colistine } \\
( \pm \text { tigécycline })\end{array}$ \\
& ou \\
& Ceftazidime-avibactam \\
& $( \pm$ méropénème $)$ \\
OXA-48 & Aztréonam \\
& ou \\
& Ceftazidime-avibactam \\
Métallo- $\beta$-carbapénémase & Colimycine + fosfomycine \\
& $( \pm$ tigécycline $)$
\end{tabular}

Tableau 2 Concentrations critiques (CMI) et diamètres critiques d'inhibition

\begin{tabular}{|c|c|c|c|c|c|}
\hline \multirow[t]{2}{*}{ Carbapénèmes } & \multicolumn{2}{|c|}{$\begin{array}{l}\text { Concentrations } \\
\text { critiques }(\mathrm{mg} / \mathrm{l})\end{array}$} & \multirow[t]{2}{*}{$\begin{array}{l}\text { Charge } \\
\text { du disque } \\
(\mu \mathrm{g})\end{array}$} & \multicolumn{2}{|c|}{$\begin{array}{l}\text { Diamètres } \\
\text { critiques } \\
(\mathrm{mm})\end{array}$} \\
\hline & $\mathbf{S} \leq$ & $\mathbf{R}>$ & & $\mathbf{S} \geq$ & $\mathbf{R}<$ \\
\hline Imipénème & 2 & 8 & 10 & 22 & 16 \\
\hline Méropénème & 2 & 8 & 10 & 22 & 16 \\
\hline
\end{tabular}

peuvent être à l'origine d'échec thérapeutique et constituent un des facteurs de risque d'émergence de résistance. Cette dernière est aussi en partie expliquée par l'utilisation croissante de la colistine, notamment en monothérapie, ces dernières années, et certains travaux évoquent un terrain génétique sous-jacent. La résistance à la colistine constitue un facteur de risque indépendant de mortalité $[21,22]$. Pour toutes ces raisons, il est important d'optimiser la prescription de la colistine à travers les différents moyens suivants. Elle doit être utilisée en association, le plus souvent avec les carbapénèmes dans le traitement des infections à EPC sensibles à la colistine et notamment dans les infections où les CMI sont au-delà de $1 \mathrm{mg} / \mathrm{l}$. En raison des sous-dosages constatés, notamment en réanimation, il est recommandé d'utiliser une dose de charge de 9 MUI de colimycine suivie d'une dose journalière de 9 MUI en l'absence d'altération de la fonction rénale $[18,23,24]$. Certains auteurs préconisent l'administration de $2 \times 4,5 \mathrm{MUI} / \mathrm{j}$ après la dose de charge, avec une surveillance de la toxicité rénale, particulièrement en cas d'administration concomitante d'autres médicaments néphrotoxiques [17,19]. La colistine peut également être administrée en aérosol à la dose de 2 MUI toutes les huit heures dans les pneumonies [25].

Pour les souches résistantes à la colistine, certains auteurs proposent une association colistine-rifampicine permettant d'avoir une activité synergique au niveau des infections à K. pneumoniae KPC. L'atteinte de l'intégrité membranaire secondaire à l'activité de la colistine permet la pénétration et secondairement l'action de la rifampicine [26,27].

Reconnue comme l'une des molécules les plus actives in vitro, la colistine est une thérapeutique de choix dans le cadre d'une association d'antibiotiques sous réserve d'une optimisation de la dose et d'une surveillance de la fonction rénale.

\section{Tigécycline}

La tigécycline, premier membre de la famille des glycylcyclines, a une affinité environ cinq fois plus puissante que les tétracyclines au niveau de la sous-unité ribosomale 30S. Antibiotique bactériostatique à large spectre avec un grand volume de distribution, elle conserve une activité sur un grand nombre de BGN multirésistants. Dans un travail publié en 2015 concernant l'activité in vitro de la tigécycline sur des souches d'EPC provenant de 18 pays européens, Sader et al. rapportent une sensibilité de 88,6\% [28]. Cette tendance est attestée par d'autres travaux [29]. Néanmoins, la tigécycline a été associée à un surrisque d'échecs cliniques et de mortalité probablement dû aux faibles concentrations sériques obtenues $(\leq 0,6 \mu \mathrm{g} / \mathrm{ml})[30]$ avec les doses recommandées dans les indications d'autorisation de mise sur le marché et à son utilisation en monothérapie dans les infections sévères [31].

Ramirez et al. [32] ont démontré dans un essai randomisé de phase 2 que l'utilisation d'un traitement par de la tigécycline à des doses plus élevées suivant deux schémas thérapeutiques (dose de charge de $150 \mathrm{mg}$ suivie de $2 \times 75 \mathrm{mg} / \mathrm{j}$ ou dose de charge de $200 \mathrm{mg}$ suivie de $2 \times 100 \mathrm{mg} / \mathrm{j}$ ) n'était pas inférieure à un traitement par imipénème/cilastatine à la dose de $1 \mathrm{~g} \times 3 / \mathrm{j}$ dans le traitement des pneumopathies nosocomiales. Le taux de succès pour ces trois schémas thérapeutiques était respectivement de 69,6, 85 et $75 \%$. Dans une stratégie d'épargne des carbapénèmes dans des infections dues à K. pneumoniae KPC en réanimation, Sbrana et al. [33] rapportent, dans une étude observationnelle avec une faible population de patients, que l'utilisation d'une forte posologie de tigécycline associée à la colistine ou à la gentamicine et à la fosfomycine dans certains cas obtenait un succès clinique dans $92 \%$ des cas.

Ainsi, la posologie de tigécycline proposée actuellement comporte une dose de charge de $200 \mathrm{mg}$ suivie d'une dose journalière de $2 \times 100 \mathrm{mg}[7,17]$. Une meilleure efficacité est observée au prix d'une majoration de la mauvaise tolérance digestive.

\section{Fosfomycine}

Agissant au niveau de la synthèse du peptidoglycane, sa faible masse moléculaire lui confère une bonne diffusion tissulaire [34]. Elle possède une activité bactéricide avec un 
large spectre vis-à-vis des bactéries à Gram positif et négatif. Concernant les souches de K. pneumoniae résistantes aux carbapénèmes, on retrouve une sensibilité allant de 39,2 à $100 \%$ dans une revue de la littérature publiée en 2016 avec néanmoins des CMI 50 et une CMI 90 plus élevées que pour les autres bactéries sensibles à cette molécule [35]. Les auteurs recommandent d'avoir une connaissance de l'écologie locale avant d'utiliser cette molécule. Bien que l'activité in vitro sur K. pneumoniae de cet antibiotique semble intéressante, peu de travaux cliniques ont été publiés [36,37]. Dans une étude multicentrique observationnelle prospective menée au sein de plusieurs services de réanimation, l'évolution clinique et microbiologique a été évaluée chez 48 patients présentant principalement des pneumopathies acquises sous ventilation mécanique et des bactériémies. La fosfomycine était administrée en i.v. à $24 \mathrm{~g} / \mathrm{j}$ pour une durée médiane de 14 jours en monothérapie $(37,5 \%$ des cas), en bithérapie $(54,2 \%$ des cas) ou en trithérapie ( $8,3 \%$ des cas) avec la colistine, la tigécycline ou la gentamicine. Le taux de succès clinique à j14 était de $54,2 \%$, avec un taux d'éradication microbiologique de 56,3\%. L'effet secondaire le plus rencontré était une hypokaliémie chez environ 20 à $25 \%$ des patients [38].

L'utilisation de la fosfomycine en monothérapie est déconseillée en raison du risque d'émergence de résistance et d'échec thérapeutique. Dans les associations proposées, on recommande une posologie de $4 \mathrm{~g} \times 6 / \mathrm{j}$ de fosfomycine, avec une surveillance rigoureuse de la kaliémie sans oublier l'apport sodique majeur dans cette option thérapeutique [17].

\section{Aminosides}

Les aminosides constituent une option thérapeutique dans les associations d'antibiotiques proposées lors de la prise en charge des infections à entérobactéries productrices de carbapénémases. Dans une étude rétrospective concernant 50 cas de sepsis, on note une diminution de la mortalité lors des infections à K. pneumoniae productrice de carbapénémases ayant été traitées par un aminoside [39]. En raison de la toxicité rénale, une association avec la colistine est fortement déconseillée, et elle sera exceptionnellement privilégiée dans les cas de résistance aux carbapénèmes. Par ailleurs, on rapporte une diminution de l'incidence de la néphrotoxicité en cas d'association avec la fosfomycine probablement secondaire à l'effet protecteur de cette dernière [40].

\section{Association d'antibiotiques}

Une revue de 20 études cliniques rapporte un taux de mortalité plus faible en cas d'association de molécules actives in vitro par rapport à une monothérapie $(27,4$ vs $38,7 \% ; p<0,001)$
[7]. Les auteurs soulignent également que le taux de mortalité demeure plus faible, $18,8 \%$, quand cette association comporte un carbapénème, alors qu'il est à $30,7 \%$ dans le cas contraire. Ces résultats en faveur d'une association thérapeutique, comportant un carbapénème, vs une monothérapie sont également retrouvés dans d'autres études $[8,9,41,42]$.

Falagas et al. [43] rapportent, après l'analyse de 22 études non randomisées comprenant 692 patients, qu'une association d'antibiotiques reste la stratégie optimale dans la prise en charge d'une infection sévère chez des patients fragiles. Néanmoins, ils rapportent sur le plan purement descriptif une mortalité variable pouvant aller jusqu'à 67,50 et $64 \%$ selon la réalisation d'une association par carbapénèmecolistine, tigécycline-gentamicine et tigécycline-colistine respectivement. Dans ce travail, l'association carbapénème-colistine était la moins bonne option. L'effet bénéfique d'une association d'antibiotiques, par rapport à une monothérapie par de la colistine, est également retrouvé dans une revue de la littérature [44].

Ces différentes données en faveur d'une association thérapeutique doivent être analysées avec prudence, car issues essentiellement d'études observationnelles comportant un effectif faible de patients [45], en attendant les résultats d'études randomisées contrôlées [46].

Dans un travail publié récemment, Fredborg et al. [47] rapportent l'activité synergique du méropénème dans une double et triple association, soulignant que l'association méropénème-polymyxine-rifampicine représentait la meilleure association synergique. Les auteurs recommandent la réalisation de tests in vitro afin de rechercher la meilleure combinaison thérapeutique.

\section{Avibactam}

Nouvel inhibiteur des $\beta$-lactamases, l'avibactam, a une activité vis-à-vis des $\beta$-lactamases à spectre élargi (BLSE), des Amp C ainsi que des carbapénémases de types KPC et Oxa48. Par contre, il reste inactif vis-à-vis des souches produisant des métallo- $\beta$-lactamases. Associé à la ceftazidime, il a obtenu en France l'autorisation de mise sur le marché dans le traitement des infections intra-abdominales compliquées, des infections urinaires compliquées dont les pyélonéphrites aiguës, des pneumonies nosocomiales dont les pneumonies acquises sous ventilation mécanique, des infections dues à des bactéries aérobies à Gram négatif chez des adultes pour qui les options thérapeutiques sont limitées. Plusieurs études de non-infériorité ont démontré son intérêt vis-à-vis du traitement comparateur dans ces différents contextes [48-51].

Concernant les carbapénémases, il n'y a pas d'étude randomisée contrôlée publiée à ce jour. La sensibilité in vitro de 961 souches d'entérobactéries non sensibles aux carbapénèmes avec une carbapénémase de type KPC ou OXA-48-like a 
été évaluée par de Jonge et al. en 2016 [52]. 98,7\% des souches porteuses d'une carbapénémase de type KPC et 98,5\% des souches porteuses d'une carbapénémase de type OXA48-like étaient sensibles à ceftazidime-avibactam.

Shields et al. [53] rapportent une efficacité supérieure de l'association ceftazidime-avibactam par rapport à d'autres associations thérapeutiques dans la prise en charge des bactériémies à $K$. pneumoniae résistantes aux carbapénèmes. Le taux de succès était respectivement de 85,48 et 40 dans les groupes traités par ceftazidime-avibactam, carbapénèmeaminoglycosides et carbapénème-colimycine. La survenue d'une incidence plus faible d'insuffisance rénale dans le groupe ceftazidime-avibactam peut représenter un atout non négligeable de cette association.

Dans un travail publié en 2016, ce même auteur signale la survenue de résistance à l'association ceftazidime-avibactam au décours d'un traitement et précise que cela mérite d'être surveillé rigoureusement [54].

Les résultats d'une étude prospective multicentrique observationnelle publiée en 2017 comparant ceftazidimeavibactam à la colimycine dans le cadre d'une association thérapeutique sont relativement encourageants. Les auteurs proposent ainsi ceftazidime-avibactam comme une alternative raisonnable à la colimycine pour le traitement des infections à $K$. pneumoniae productrice de carbapénémases [55].

L'intérêt de cette association dans ce contexte est aussi souligné dans différentes publications de séries de cas cliniques [56,57].

Enfin, l'association ceftazidime-avibactam n'ayant aucune activité au niveau des métallo- $\beta$-lactamases, certains auteurs ont traité des infections secondaires à ce mécanisme de résistance par une association ceftazidime-avibactam et aztréonam. Les résultats relativement encourageants doivent être confirmés par des études supplémentaires [58,59].

Les résultats provenant de ces différentes études semblent prometteurs pour l'association ceftazidime-avibactam et doivent être confirmés par des études randomisées contrôlées afin d'évaluer le bénéfice, la toxicité ainsi que les résistances induites.

\section{Perspectives thérapeutiques [60,61]}

Quelques molécules et inhibiteurs des $\beta$-lactamases tels que l'éravacycline, la plazomycine, le relebactam et le vaborbactam sont en cours d'essai et pourront représenter les perspectives thérapeutiques.

\section{Proposition thérapeutique}

Les possibilités thérapeutiques ne sont pas nombreuses, et l'utilisation des associations paraît nécessaire pour les infec- tions sévères. Les données disponibles dans la littérature quant à l'efficacité des combinaisons possibles peuvent être discutables, car issues, pour la grande majorité, d'études rétrospectives comportant de faibles effectifs et incluant des patients infectés par des bactéries portant un mécanisme prédominant de type KPC. Nous proposons ci-après un arbre thérapeutique (Fig. 1) ainsi qu'un schéma d'antibiothérapie en fonction du type de carbapénémase (Tableau 1). Par ailleurs, il est important de noter que si cette distinction en différentes classes est importante en théorie, elle pourrait en pratique ne pas permettre de recourir aux antibiotiques théoriquement épargnés du fait des mécanismes de résistance associés [62]. Néanmoins, il reste primordial d'anticiper le risque chez les patients colonisés et de solliciter le laboratoire de bactériologie afin de tester les associations possibles avant la survenue de l'infection.

\section{Conclusion}

Dans l'attente de l'apport des futures études et options thérapeutiques, les stratégies actuelles limitées ont redonné un nouvel intérêt à certaines « vieilles molécules » et font appel à des associations d'antibiotiques avec une optimisation de leurs modalités d'administration. Des essais contrôlés randomisés sont plus que nécessaires, et la maîtrise du bon usage des antibiotiques reste toujours d'actualité.

Liens d'intérêts : Jean-Ralph Zahar : laboratoires MSD et Pfizer et intervention comme orateur dans des symposiums.

Joy Y. Mootien : laboratoires MSD et Pfizer.

\section{Références}

1. Tamma PD, Goodman KE, Harris AD, Tekle T, Roberts A, Taiwo A, Simner PJ, (2017) Comparing the outcomes of patients with carbapenemase-producing and non-carbapenemase-producing carbapenem-resistant Enterobacteriaceae bacteremia. Clin Infect Dis 64: $257-264$

2. Kassis-Chikhani N, Saliba F, Carbonne A, Neuville S, Decre D, Sengelin C, Guerin C, Gastiaburu N, Lavigne-Kriaa A, Boutelier C, Arlet G, Samuel D, Castaing D, Dussaix E, Jarlier V, (2010) Extended measures for controlling an outbreak of VIM-1 producing imipenem-resistant Klebsiella pneumoniae in a liver transplant centre in France, 2003-2004. Euro Surveill 15: 19713

3. http://invs.santepubliquefrance.fr/Dossiers-thematiques/Maladiesinfectieuses/Infections-associees-aux-soins/Surveillance-desinfections-associees-aux-soins-IAS/Enterobacteries-productricesde-carbapenemases-EPC/Episodes-impliquant-des-EPC-enFrance.-Situation-epidemiologique-du-31-decembre-2015

4. Société Française de Microbiologie tableaux des concentrations critiques pour l'interprétation des CMI et des diamètres des zones d'inhibition. In : CASFM : EUCAST : Société Française de Microbiologie Ed ; 2017 : 38 
5. Daikos GL, Markogiannakis A, (2011) Carbapenemaseproducing Klebsiella pneumoniae: (when) might we still consider treating with carbapenems? Clin Microbiol Infect 17: 1135-1141

6. Tumbarello M, Viale P, Viscoli C, Trecarichi EM, Tumietto F, Marchese A, Spanu T, Ambretti S, Ginocchio F, Cristini F, Losito AR, Tedeschi S, Cauda R, Bassetti M, (2012) Predictors of mortality in bloodstream infections caused by Klebsiella pneumoniae carbapenemase-producing $K$. pneumoniae: importance of combination therapy. Clin Infect Dis 55: 943-950

7. Tzouvelekis LS, Markogiannakis A, Piperaki E, Souli M, Daikos GL, (2014) Treating infections caused by carbapenemaseproducing Enterobacteriaceae. Clin Microbiol Infect 20: 862-872

8. Daikos GL, Tsaousi S, Tzouvelekis LS, Anyfantis I, Psichogiou M, Argyropoulou A, Stefanou I, Sypsa V, Miriagou V, Nepka M, Georgiadou S, Markogiannakis A, Goukos D, Skoutelis A, (2014) Carbapenemase-producing Klebsiella pneumoniae bloodstream infections: lowering mortality by antibiotic combination schemes and the role of carbapenems. Antimicrob Agents Chemother 58: 2322-2328

9. Tumbarello M, Trecarichi EM, De Rosa FG, Giannella M, Giacobbe DR, Bassetti M, Losito AR, Bartoletti M, Del Bono V, Corcione S, Maiuro G, Tedeschi S, Celani L, Cardellino CS, Spanu T, Marchese A, Ambretti S, Cauda R, Viscoli C, Viale P; ISGRI-SITA (Italian Study Group on Resistant Infections of the Società Italiana Terapia Antinfettiva), (2015) Infections caused by KPC-producing Klebsiella pneumoniae: differences in therapy and mortality in a multicentre study. J Antimicrob Chemother 70: 2133-2143

10. Tzouvelekis LS, Markogiannakis A, Psichogiou M, Tassios PT, Daikos GL, (2012) Carbapenemases in Klebsiella pneumoniae and other Enterobacteriaceae: an evolving crisis of global dimensions. Clin Microbiol Rev 25: 682-707

11. Cattoir V, (2014) Traitement des infections dues à entérobactéries productrices de carbapénémases. J Anti Infect 16: 99-105

12. Wiskirchen DE, Crandon JL, Nicolau DP, (2013) Impact of various conditions on the efficacy of dual carbapenem therapy against KPC-producing Klebsiella pneumoniae. Int J Antimicrob Agents 41: 582-585

13. Giamarellou H, Galani L, Baziaka F, Karaiskos I, (2013) Effectiveness of a double-carbapenem regimen for infections in humans due to carbapenemase-producing pandrug-resistant Klebsiella pneumoniae. Antimicrob Agents Chemother 57: 2388-2390

14. Oliva A, D'Abramo A, D'Agostino C, Iannetta M, Mascellino MT, Gallinelli C, Mastroianni CM, Vullo V, (2014) Synergistic activity and effectiveness of a double-carbapenem regimen in pandrug-resistant Klebsiella pneumoniae bloodstream infections. J Antimicrob Chemother 69: 1718-1720

15. Poirel L, Kieffer N, Nordmann P, (2016) In vitro evaluation of dual carbapenem combinations against carbapenemase-producing Enterobacteriaceae. J Antimicrob Chemother 71: 156-161

16. Cprek JB, Gallagher JC, (2015) Ertapenem-containing doublecarbapenem therapy for treatment of infections caused by carbapenem-resistant Klebsiella pneumoniae. Antimicrob Agents Chemother 60: 669-673

17. Bassetti M, Peghin M, Pecori D, (2016) The management of multidrug-resistant Enterobacteriaceae. Curr Opin Infect Dis 29: 583-594

18. Plachouras D, Karvanen M, Friberg LE, Papadomichelakis E, Antoniadou A, Tsangaris I, Karaiskos I, Poulakou G, Kontopidou F, Armaganidis A, Cars O, Giamarellou H, (2009) Population pharmacokinetic analysis of colistin methanesulfonate and colistin after intravenous administration in critically ill patients with infections caused by gram-negative bacteria. Antimicrob Agents Chemother 53: 3430-3436

19. Rocco M, Montini L, Alessandri E, Venditti M, Laderchi A, De Pascale G, Raponi GG, Vitale M, Pietropaoli P, Antonelli M,
(2013) Risk factors for acute kidney injury in critically ill patients receiving high intravenous doses of colistin methanesulfonate and/or other nephrotoxic antibiotics: a retrospective cohort study. Crit Care 17: R174

20. Kim J, Lee KH, Yoo S, Pai H, (2009) Clinical characteristics and risk factors of colistin-induced nephrotoxicity. Int J Antimicrob Agents 34: 434-438

21. Giacobbe DR, Del Bono V, Trecarichi EM, De Rosa FG, Giannella M, Bassetti M, Bartoloni A, Losito AR, Corcione S, Bartoletti M, Mantengoli E, Saffioti C, Pagani N, Tedeschi S, Spanu T, Rossolini GM, Marchese A, Ambretti S, Cauda R, Viale P, Viscoli C, Tumbarello M; ISGRI-SITA (Italian Study Group on Resistant Infections of the Società Italiana Terapia Antinfettiva), (2015) Risk factors for bloodstream infections due to colistinresistant KPC-producing Klebsiella pneumoniae: results from a multicenter case-control-control study. Clin Microbiol Infect 2: 1106.e1-1106.e8

22. Wright MS, Suzuki Y, Jones MB, Marshall SH, Rudin SD, Van Duin D, Kaye K, Jacobs MR, Bonomo RA, Adams MD, (2015) Genomic and transcriptomic analyses of colistin-resistant clinical isolates of Klebsiella pneumoniae reveal multiple pathways of resistance. Antimicrob Agents Chemother 59: 536-543

23. Mohamed AF, Karaiskos I, Plachouras D, Karvanen M, Pontikis K, Jansson B, Papadomichelakis E, Antoniadou A, Giamarellou $\mathrm{H}$, Armaganidis A, Cars O, Friberg LE, (2012) Application of a loading dose of colistin methanesulfonate in critically ill patients: population pharmacokinetics, protein binding, and prediction of bacterial kill. Antimicrob Agents Chemother 56: 4241-4249

24. Dalfino L, Puntillo F, Mosca A, Monno R, Spada ML, Coppolecchia S, Miragliotta G, Bruno F, Brienza N, (2012) High-dose, extended-interval colistin administration in critically ill patients: is this the right dosing strategy? A preliminary study. Clin Infect Dis 54: $1720-1726$

25. Florescu DF, Qiu F, McCartan MA, Mindru C, Fey PD, Kalil AC, (2012) What is the efficacy and safety of colistin for the treatment of ventilator-associated pneumonia? A systematic review and meta-regression. Clin Infect Dis 54: 670-680

26. Tascini C, Tagliaferri E, Giani T, Leonildi A, Flammini S, Casini B, Lewis R, Ferranti S, Rossolini GM, Menichetti F, (2013) Synergistic activity of colistin plus rifampin against colistinresistant KPC-producing Klebsiella pneumoniae. Antimicrob Agents Chemother 57: 3990-3993

27. Forrest GN, Tamura K, (2010) Rifampin combination therapy for non mycobacterial infections. Clin Microbiol Rev 23: 14-34

28. Sader HS, Castanheira M, Flamm RK, Mendes RE, Farrell DJ, Jones RN, (2015) Tigecycline activity tested against carbapenem-resistant Enterobacteriaceae from 18 European nations: results from the SENTRY surveillance program (20102013). Diagn Microbiol Infect Dis 83: 183-186

29. Ceccarelli G, Falcone M, Giordano A, Mezzatesta ML, Caio C, Stefani S, Venditti M, (2013) Successful ertapenem-doripenem combination treatment of bacteremic ventilator-associated Pneumonia due to colistin-resistant KPC-producing Klebsiella pneumoniae. Antimicrob Agents Chemother 57: 2900-2901

30. Freire AT, Melnyk V, Kim MJ, Datsenko O, Dzyublik O, Glumcher F, Chuang YC, Maroko RT, Dukart G, Cooper CA, KorthBradley JM, Dartois N, Gandjini H, (2010) Comparison of tigecycline withimipenem/cilastatin for the treatment of hospitalacquired pneumonia. Diagn Microbiol Infect Dis 68: 140-151

31. Poulakou G, Kontopidou FV, Paramythiotou E, Kompoti M, Katsiari M, Mainas E, Nicolaou C, Yphantis D, Antoniadou A, Trikka-Graphakos E, Roussou Z, Clouva P, Maguina N, Kanellakopoulou K, Armaganidis A, Giamarellou H, (2009) Tigecycline in the treatment of infections from multidrug resistant gramnegative pathogens. J Infect 58: 273-284 
32. Ramirez J, Dartois N, Gandjini H, Yan JL, Korth-Bradley J, McGover PC, (2013) Randomized phase 2 trial to evaluate the clinical efficacy of two high-dosage tigecycline regimens versus imipenemcilastatin for treatment of hospital acquired Pneumonia. Antimicrob Agents Chemother 57: 1756-1762

33. Sbrana F, Malacarne P, Viaggi B, Costanzo S, Leonetti P, Leonildi A, Casini B, Tascini C, Menichetti F, (2013) Carbapenemsparing antibiotic regimens for infections caused by Klebsiella pneumoniae carbapenemase-producing $K$. pneumoniae in intensive care unit. Clin Infect Dis 56: 697-700

34. Raz R, (2012) Fosfomycin: an old-new antibiotic. Clin Microbiol Infect 18: 4-7

35. Vardakas KZ, Legakis NJ, Triarides N, Falagas ME, (2016) Susceptibility of contemporary isolates to fosfomycin: a systematic review of the literature. Int J Antimicrob Agents 47: 269-285

36. Albiero J, Sy SK, Mazucheli J, Caparroz-Assef SM, Costa BB, Alves JL, Gales AC, Tognim MC, (2016) Pharmacodynamic evaluation of the potential clinical utility of fosfomycin and meropenem in combination therapy against KPC-2-producing Klebsiella pneumoniae. Antimicrob Agents Chemother 60: 4128-4139

37. Rizek C, Ferraz JR, van der Heijden IM, Giudice M, Mostachio AK, Paez J, Carrilho C, Levin AS, Costa SF, (2015) In vitro activity of potential old and new drugs against multidrug-resistant gram-negatives. J Infect Chemother 21: 114-117

38. Pontikis K, Karaiskos I, Bastani S, Dimopoulos G, Kalogirou M, Katsiari M, Oikonomou A, Poulakou G, Roilides E, Giamarellou $\mathrm{H}$, (2014) Outcomes of critically ill intensive care unit patients treated with fosfomycin for infections due to pandrug-resistant and extensively drug-resistant carbapenemase-producing Gramnegative bacteria. Int J Antimicrob Agents 43: 52-59

39. Gonzalez-Padilla M, Torre-Cisneros J, Rivera-Espinar F, PontesMoreno A, López-Cerero L, Pascual A, Natera C, Rodríguez M, Salcedo I, Rodríguez-López F, Rivero A, Rodríguez-Baño J, (2015) Gentamicin therapy for sepsis due to carbapenemresistant and colistin-resistant Klebsiella pneumoniae. J Antimicrob Chemother 70: 905-913

40. Morin JP, Olier B, Viotte G, Fillastre JP, (1984) Can fosfomycin reduce the nephrotoxicity of aminoglycosides? Pathol Biol 32: 338-342

41. Doi Y, Paterson DL, (2015) Carbapenemase-producing Enterobacteriaceae. Semin Respir Crit Care Med 36: 74-84

42. Qureshi ZA, Paterson DL, Potoski BA, Kilayko MC, Sandovsky G, Sordillo E, Polsky B, Adams-Haduch JM, Doi Y, (2012) Treatment outcome of bacteremia due to KPC-producing Klebsiella pneumoniae: superiority of combination antimicrobial regimens. Antimicrob Agents Chemother 56: 2108-2113

43. Falagas ME, Lourida P, Poulikakos P, Rafailidis PI, Tansarli GS, (2014) Antibiotic treatment of infections due to carbapenemresistant Enterobacteriaceae: systematic evaluation of the available evidence. Antimicrob Agents Chemother 58: 654-663

44. Lee GC, Burgess DS, (2012) Treatment of Klebsiella pneumoniae carbapenemase (KPC) infections: a review of published case series and case reports. Ann Clin Microbiol Antimicrob 11: 32

45. Paul M, Carmeli Y, Durante-Mangoni E, Mouton JW, Tacconelli E, Theuretzbacher U, Mussini C, Leibovici L, (2014) Combination therapy for carbapenem-resistant Gram-negative bacteria. J Antimicrob Chemother 69: 2305-2309

46. Dickstein Y, Leibovici L, Yahav D, Eliakim-Raz N, Daikos GL, Skiada A, Antoniadou A, Carmeli Y, Nutman A, Levi I, Adler A, Durante-Mangoni E, Andini R, Cavezza G, Mouton JW, Wijma RA, Theuretzbacher U, Friberg LE, Kristoffersson AN, Zusman O, Koppel F, Benattar YD, Altunin S, Paul M; The AIDA Consortium, (2016) Multicentre open-label randomised controlled trial to compare colistin alone with colistin plus meropenem for the treatment of severe infections caused by carbapenem- resistant Gram-negative infections (AIDA): a study protocol. BMJ Open 6: e009956

47. Fredborg M, Sondergaard TE, Wang M, (2017) Synergistic activities of meropenem double and triple combinations against carbapenemase-producing Enterobacteriaceae. Diagn Microbiol Infect Dis 88: $355-360$

48. Torres A, Zhong N, Pachl J, Timsit JF, Kollef M, Chen Z, Song J, Taylor D, Laud PJ, Stone GG, Chow JW, (2018) Ceftazidimeavibactam versus meropenem in nosocomial pneumonia, including ventilator-associated pneumonia (REPROVE): a randomised, double-blind, phase 3 non-inferiority trial. Lancet Infect Dis 18: $285-295$

49. Carmeli Y, Armstrong J, Laud PJ, Newell P, Stone G, Wardman A, Gasink LB, (2016) Ceftazidime-avibactam or best available therapy in patients with ceftazidime-resistant Enterobacteriaceae and Pseudomonas aeruginosa complicated urinary tract infections or complicated intra-abdominal infections (REPRISE): a randomised, pathogen-directed, phase 3 study. Lancet Infect Dis 16: 661-673

50. Mazuski JE, Gasink LB, Armstrong J, Broadhurst H, Stone GG, Rank D, Llorens L, Newell P, Pachl J, (2016) Efficacy and safety of ceftazidime-avibactam plus metronidazole versus meropenem in the treatment of complicated intra-abdominal infection: results from a randomized, controlled, double-blind, phase 3 program. Clin Infect Dis 62: 1380-1389

51. Wagenlehner FM, Sobel JD, Newell P, Armstrong J, Huang X, Stone GG, Yates K, Gasink LB, (2016) Ceftazidime-avibactam versus doripenem for the treatment of complicated urinary tract infections, including acute pyelonephritis: RECAPTURE, a phase 3 randomized trial program. Clin Infect Dis 63: 754-762

52. de Jonge BL, Karlowsky JA, Kazmierczak KM, Biedenbach DJ, Sahm DF, Nichols WW, (2016) In vitro susceptibility to ceftazidime-avibactam of carbapenem-nonsusceptible Enterobacteriaceae isolates collected during the INFORM global surveillance study (2012 to 2014). Antimicrob Agents Chemother 60: 3163-3169

53. Shields RK, Nguyen MH, Chen L, Press EG, Potoski BA, Marini RV, Doi Y, Kreiswirth BN, Clancy CJ, (2017) Ceftazidimeavibactam is superior to other treatment regimens against carbapenem-resistant Klebsiella pneumoniae bacteremia. Antimicrob Agents Chemother 61(8): e00883-17

54. Shields RK, Potoski BA, Haidar G, Hao B, Doi Y, Chen L, Press EG, Kreiswirth BN, Clancy CJ, Nguyen MH, (2016) Clinical outcomes, drug toxicity, and emergence of ceftazidime-avibactam resistance among patients treated for carbapenem-resistant Enterobacteriaceae infections. Clin Infect Dis 63: 1615-1618

55. van Duin D, Lok JJ, Earley M, Cober E, Richter SS, Perez F, Salata RA, Kalayjian RC, Watkins RR, Doi Y, Kaye KS, Fowler VG Jr, Paterson DL, Bonomo RA, Evans S; Antibacterial Resistance Leadership Group, (2018) Colistin versus ceftazidimeavibactam in the treatment of infections due to carbapenemresistant Enterobacteriaceae. Clinical Infect Dis 66: 163-171

56. Temkin E, Torre-Cisneros J, Beovic B, Benito N, Giannella M, Gilarranz R, Jeremiah C, Loeches B, Machuca I, Jiménez-Martín MJ, Martínez JA, Mora-Rillo M, Navas E, Osthoff M, Pozo JC, Ramos JC, Rodriguez M, Sánchez-García M, Viale P, Wolff M, Carmeli Y, (2017) Ceftazidime-avibactam as salvage therapy for infections caused by carbapenem-resistant organisms. Antimicrob Agents Chemother 61(2): e01964-16

57. Gugliandolo A, Caio C, Mezzatesta ML, Rifici C, Bramanti P, Stefani S, Mazzon E, (2017) Successful ceftazidime-avibactam treatment of MDR-KPC-positive Klebsiella pneumoniae infection in a patient with traumatic brain injury: a case report. Medicine 96: e7664

58. Davido B, Fellous L, Lawrence C, Maxime V, Rottman M, Dinh A, (2017) Ceftazidime-avibactam and aztreonam, an interesting strategy to overcome $\beta$-lactam resistance conferred by metallo$\beta$-lactamases in Enterobacteriaceae and Pseudomonas aeruginosa. Antimicrob Agents Chemother 61: e01008-17 
59. Marshall S, Hujer AM, Rojas LJ, Papp-Wallace KM, Humphries RM, Spellberg B, Hujer KM, Marshall EK, Rudin SD, Perez F, Wilson BM, Wasserman RB, Chikowski L, Paterson DL, Vila AJ, van Duin D, Kreiswirth BN, Chambers HF, Fowler VG Jr, Jacobs MR, Pulse ME, Weiss WJ, Bonomo RA, (2017) Can ceftazidimeavibactam and aztreonam overcome $\beta$-lactam resistance conferred by metallo- $\beta$-lactamases in Enterobacteriaceae? Antimicrob Agents Chemother 61(4): e02243-16
60. Bowers DR, Huang V, (2016) Emerging issues and treatment strategies in carbapenem-resistant Enterobacteriaceae (CRE). Curr Infect Dis Rep 18: 48

61. Karaiskos I, Giamarellou H, (2014) Multidrug-resistant and extensively drug-resistant Gram-negative pathogens: current and emerging therapeutic approaches. Expert Opin Pharmacother 15: 1351-1370

62. Nordmann P, Dortet L, Poirel L, (2012) Carbapenem-resistance in Enterobacteriaceae: here is the storm! Trends Mol Med 18: 263-272 